\title{
Current Some Day Smoker
}

National Cancer Institute

\section{Source}

National Cancer Institute. Current Some Day Smoker. NCI Thesaurus. Code C67146.

Indicates a person who has smoked at least 100 cigarettes in his or her lifetime, who smokes now, but does not smoke every day. 